\title{
画HAD
}

ISSN-L: 2530-5115

DOI: http://doi.org/10.22585/hospdomic.v2i4.51

\section{La Bibliometría en la evaluación de la actividad científica}

\section{Bibliometrics in the evaluation of scientific activity}

\author{
Vicente Tomás-Górriz' , Vicente Tomás-Casterá ${ }^{2}$ \\ 1. Universidad de Valencia, Departamento de Toxicología, Burjassot, España. \\ 2. Centro de Salud Pública de la Conselleria de Sanidad Universal y Salud Pública, Alzira, España.
}

Correspondencia/Correspondence

Vicente Tomás-Górriz

Universidad de Valencia, Departamento de

Toxicología, Burjassot, España

vitogo91@gmail.com

Recibido/Received

19.07.2018

Aceptado/Accepted

01.09 .2018
Conflicto de Intereses/Competing interest Los autores declaran la inexistencia de conflicto de interés

CÓMO CITAR ESTE TRABAJO | HOW TO CITE THIS PAPER

Tomás-Górriz V, Tomás-Casterá V. La Bibliometría en la evaluación de la actividad científica. Hosp Domic. 2018;2(4):145-63. 


\section{RESUMEN}

El análisis y la evaluación de la información y el conocimiento resultante de la actividad científica es un elemento imprescindible para todos los programas de investigación pública, tecnología y desarrollo que se implementan en una sociedad; y es allí donde la Ciencia de la Información brinda una ayuda inestimable, al desarrollar técnicas e instrumentos para medir la producción de conocimiento y su transformación en bienes.

La cuantificación de las publicaciones es el elemento básico de la mayor parte de los estudios bibliométricos, mientras que la de las citas puede usarse directamente como medida de la repercusión o importancia de una publicación, o de las publicaciones de una persona, institución o país. Las referencias y las citas se convierten, de esta manera, en la materia prima de la Bibliometría. Sin embargo, deben tratarse con cuidado. La cuantificación de publicaciones no está libre de sesgos.

El presente trabajo hace un análisis de las diferentes aproximaciones a la evaluación de la investigación desde la perspectiva cienciométrica. $Y$, en consecuencia, dadas las repercusiones que las evaluaciones bibliométricas tienen sobre la asignación de fondos para la investigación e incluso la acreditación y promoción profesional de los investigadores, es necesario conocer muy bien las particularidades y las limitaciones que su uso conlleva.

Palabras clave: Bibliometría; Indicadores Bibliométricos; Sistemas de Créditos y Evaluación de Investigadores; Edición; Investigación.

\section{ABSTRACT}

The analysis and assessment of information and knowledge resulting of scientific activity is an essential for all public research, technology and development programs which are implemented in a society; and this is where the Science of Information provides an invaluable help, by developing techniques and instruments to measure the production of knowledge and its transformation into goods.

The quantification of publications is the basic element of most of the bibliometric studies, meanwhile the one of the quotations can be used directly as a measure of the repercussion or importance of a publication, or of the publications of a person, institution or country. The reference and quotations become, in this way, in the raw material of the bibliometrics. However, they must be treated with care. The quantification of publications is not bias-free.

This paper analyses the different approaches to the assessment of research from a sciencemetric perspective. And, in consequence, given the repercussions that bibliometric evaluations have on the allocation of funds for research and even the accreditation and professional promotion of researchers, it is necessary to know very well the particularities and limitations that its use entails.

Keywords: Bibliometrics; Bibliometric Indicators; Researcher Performance Evaluation Systems; Publishing; Research. 
El presente artículo se fundamenta en los antecedentes de la tesis doctoral defendida en la Universidad de Alicante en el año 2013 (1).

El análisis y la evaluación de la información y el conocimiento resultante de la actividad científica es un elemento imprescindible para todos los programas de investigación pública, tecnología y desarrollo que se implementan en una sociedad; y es allí donde la Ciencia de la Información brinda una ayuda inestimable, al desarrollar técnicas e instrumentos para medir la producción de conocimiento y su transformación en bienes.

Las disciplinas métricas de la información (Bibliometría, Cienciometría e Informetría) han permitido el desarrollo de indicadores que, al margen de ventajas y limitaciones ampliamente debatidas, y sobre todo cuando son producto de un análisis multifactorial del contexto donde son aplicados, constituyen herramientas clave en la gestión de la política científica y tecnológica, y en los procesos de toma de decisiones estratégicas.

La Bibliometría como disciplina, ha tenido desde su nacimiento básicamente dos grandes ámbitos de desarrollo y aplicación; por una parte, el estudio de la ciencia y la evaluación de la producción científica y, por otra, la gestión editorial. Su desarrollo se debe a aportaciones tanto de uno como de otro medio, si bien, su consolidación definitiva ha venido de la mano del estudio de la ciencia y de la comunicación científica, sobretodo impulsado por el Institute for Scientific Information (ISI) de Filadelfia, con la publicación (en 2018 accesible on-line y facilitado por Clarivate Analytics) del Science Citation Index y con los importantes trabajos de fundamentación teórica de Price y Garfield (2-5).

Los trabajos de Price en torno a la "Ciencia de la Ciencia" (6), significaron la consolidación definitiva de la disciplina tal y como hoy se conoce. Price afirma que la finalidad es "identificar las leyes y las regularidades que rigen la actividad científica en su globalidad".

Desde su origen hasta la actualidad, la Bibliometría ha estado ligada estrechamente tanto con la Bibliografía como con las Fuentes de Información. Esta relación constituye una dependencia de estas últimas disciplinas, ya que los estudios bibliométricos se llevan a cabo a partir de los análisis de las Fuentes de Información, que constituyen la base sobre la cual se mide la producción científica. Incluso los propios investigadores de esta disciplina hacen referencia a las Fuentes de Información como el medio sobre el cual se aplican las técnicas bibliométricas que a su vez están estrechamente relacionadas con los métodos estadísticos.

Los estudios bibliométricos tienen por objeto el tratamiento y análisis cuantitativo de las publicaciones científicas, constituyendo en la actualidad la herramienta esencial para el conocimiento de la actividad investigadora, aportando datos sobre la situación científica de un país o tema de investigación, permitiendo evaluar el rendimiento de la actividad científica y su impacto en la comunidad.

Forman parte de los "estudios sociales de la ciencia" y entre sus principales aplicaciones se encuentra el área de la política científica. Estos estudios complementan de manera eficaz las opiniones y los juicios emitidos por los expertos de cada área proporcionando herramientas útiles y objetivas en los procesos de evaluación de los resultados de la actividad científica. Sin embargo, dadas las repercusiones que estas evaluaciones tienen sobre la asignación de fondos para la investigación e, incluso, la promoción profesional de los investigadores, es necesario conocer bien las características de los indicadores.

La cuantificación de las publicaciones es el elemento básico de la mayor parte de los estudios bibliométricos, mientras que la de las citas puede usarse directamente como medida de la repercusión o importancia de una publicación, o de las publicaciones de una persona, institución o país. Las referencias y las citas se convierten, de esta manera, en la materia prima de la Bibliometría. Sin embargo, deben tratarse con cuidado. La cuantificación de publicaciones no está libre de sesgos; algunos de ellos son los siguientes: 
- No reflejan la calidad de los documentos.

- Cada artículo contribuye de manera diferente al avance de la ciencia.

- Los estilos y categorías varían de acuerdo con la disciplina.

Cualquier estudio sobre Bibliometría debe perseguir dos objetivos básicos:

1. Analizar el tamaño, crecimiento y distribución de la bibliografía científica.

2. Estudiar la estructura social de los grupos que la producen y utilizan.

La importancia de que los estudios bibliométricos complementen ambos objetivos radica en la necesidad de que los datos numéricos de tamaño, crecimiento, etc., sean interpretados dentro del contexto de la comunidad científica que genera dicha producción científica.

\section{LEYES BIBLIOMÉTRICAS}

Como se ha comentado anteriormente, la Bibliometría es una parte de la Cienciometría que aplica métodos matemáticos y estadísticos a toda la literatura de carácter científico y a los autores que la producen, con el objetivo de estudiar y analizar la actividad científica. Para ello se ayuda de leyes bibliométricas, basadas en el comportamiento estadístico regular que a lo largo del tiempo han mostrado los diferentes elementos que forman parte de la ciencia.

\section{Ley de crecimiento exponencial:}

Derek J. de Solla Price constató que el crecimiento de la información científica se produce a un ritmo muy superior respecto de otros fenómenos sociales, pero muy similar a otros fenómenos observables en contextos naturales, como los procesos biológicos. Dicho crecimiento es tal que cada 15 a 30 años la información existente se duplica con un crecimiento exponencial, aunque esto depende en gran medida del área de conocimiento de la que se trate. A su vez, propuso varias etapas (ver figura 1): una fase de crecimiento exponencial propiamente dicha, en la que la tasa de crecimiento es proporcional al tamaño de la muestra, y una fase de crecimiento lineal, en la que la tasa de crecimiento es constante o independiente del tamaño del sistema. Las etapas son las siguientes:

- Fase 1: precursores

- Fase 2: crecimiento exponencial

- Fase 3: crecimiento lineal

- Fase 4: colapso del campo científico (saturación)

Sin embargo, no solo la literatura científica crece de forma exponencial, sino también el número de investigadores, por lo que la primera conclusión que obtuvo Price del crecimiento exponencial fue la contemporaneidad de la ciencia, expresión que refleja el fenómeno que consiste en que el número de científicos en la actualidad constituyen casi el total de todos los que han existido en el pasado más los actuales, siendo el número de científicos del pasado una proporción casi irrelevante frente a este número actual. No obstante, un crecimiento exponencial no puede mantenerse hasta el infinito, es decir, ha de existir un límite llamado techo o límite de saturación; admitido este límite, Price postuló que el crecimiento de la ciencia tiene forma de curva logística. 
Figura 1. Ley crecimiento exponencial de la ciencia (D.J.S. Price)

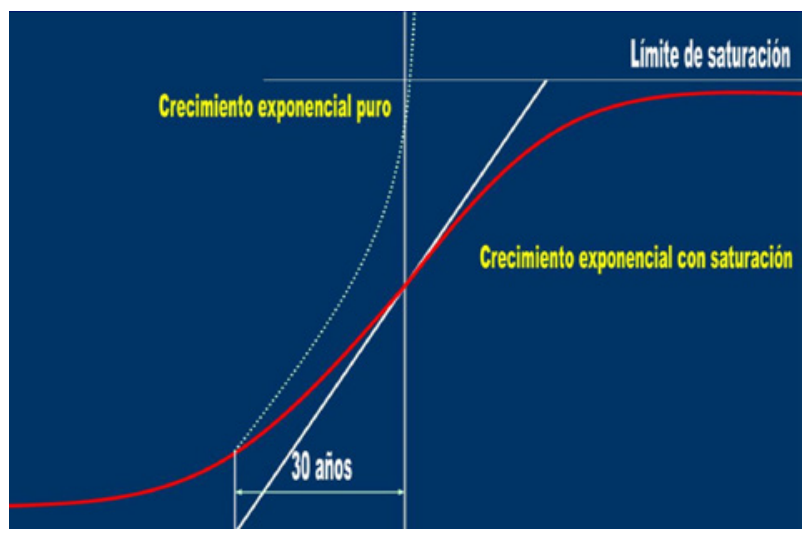

\section{Ley de la productividad de los autores:}

Lotka demostró que la relación trabajos/autor sigue un comportamiento constante bajo determinadas circunstancias. Esta ley determina que partiendo de un número de autores con un solo trabajo sobre un tema determinado, es posible predecir el número de autores con 'n' trabajos mediante la siguiente fórmula $[A(n)=K / n 2]$, ver figura 2 .

Figura 2. Ley de la productividad de los autores

- Ejemplo: si en una determinada área de conocimiento existen 100 autores que han publicado un solo trabajo (una sola firma), cuantos autores habrán publicado más trabajos.

$$
\begin{gathered}
A(n)=A(j) / n^{2} \\
n=2 \rightarrow A(2)=A(100) / 2^{2} ; A(2)=100 / 4=25 \text { autores } \\
n=10 \rightarrow A(10)=A(100) / 10^{2} ; A(10)=100 / 100=1 \text { autor }
\end{gathered}
$$

Sin embargo, no solo se trata de analizar el número de autores de un periodo determinado, sino de localizar a los autores más productivos. Es decir, habla de la relación cuantitativa entre los autores y los artículos producidos en un campo dado y en un periodo de tiempo determinado. Se concluye que a medida que aumente el número de trabajos, el número de autores disminuirá.

Es patente que la concentración de la producción científica en torno a unos pocos investigadores se agudiza a medida que se avanza en la carrera investigadora. Esto puede deberse a la existencia otros factores que, al margen del talento, contribuyen a hacer más productivos a quienes experimentan una serie de vivencias a lo largo de sus carreras profesionales que les permiten formar parte de las élites que lideran la estructura social de la Ciencia. La carrera investigadora puede tener un efecto relevante sobre las opciones de éxito profesional de los investigadores. Dicho 
efecto, conocido como 'ventaja acumulativa" o "efecto Mateo", contribuiría a agudizar la desigual distribución de la productividad y el impacto entre los investigadores; ver figura 3.

Figura 3. Efecto mateo o de ventaja acumulativa

\section{Índice de Productividad de los autores \\ La productividad sigue el conocido "efecto Mateo" o ventaja acumulativa.}

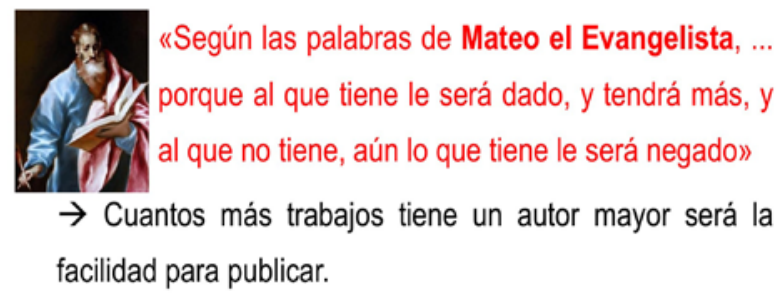

\section{Ley del envejecimiento u obsolescencia de la literatura científica:}

Price constató que la literatura científica pierde actualidad cada vez más rápidamente, estudiando por años la distribución de las referencias bibliográficas en distintas especialidades, observó que, mientras que el número de publicaciones se multiplica por dos cada 10 a 15 años, el número de citas que reciben tales publicaciones se divide en dos cada trece años aproximadamente, y estableció un cálculo para conocer el porcentaje de artículos publicados en los últimos 5 años (Índice de Price $=$ porcentaje de referencias con edad menor de 5 años).

Por otro lado, para medir el envejecimiento, Burton y Kebler idearon el concepto de semiperiodo, que se refiere al tiempo en que ha sido publicada la mitad de la literatura referenciada dentro de una disciplina científica (Semiperiodo de Burton-Kebler = Mediana de la distribución del conjunto de las referencias ordenadas por su antigüedad). El semiperiodo o vida media de la literatura de las diversas ramas científicas es variable; así, en el siglo XXI, en Ingeniería Física, es de tres a cinco años; en Química y Fisiología, de siete a ocho; en Matemáticas y Botánica, de diez años; y en Ciencias de la Salud entre siete y once años.

\section{Ley de dispersión de la literatura científica:}

Esta ley se ocupa del estudio de la dispersión de la literatura científica. Bradford realizó numerosos estudios estadísticos y obtuvo la conclusión de que existe un número de trabajos científicos sobre un tema determinado concentrado en un reducido número de revistas, las cuales a su vez, pueden distribuirse en varias zonas concéntricas de productividad decreciente. Es decir, si consultamos la literatura especializada observamos que existe un número de trabajos (aproximadamente un tercio de los existentes) agrupados en un pequeño número de revistas llamado "núcleo"; ver figura 4. 
Figura 4. Esquema de la Ley de la dispersión (anillos de Bradford)

\section{Factor de impacto}

- Indicador que mide la importancia relativa de una revista dentro de su área temática.

- Se calcula para un año determinado a partir de los datos de los dos años inmediatamente anteriores.

\begin{tabular}{|c|c|}
\hline $\begin{array}{c}\text { Factor de impacto para } \\
\text { una revista en un año } \\
\text { determinado }\end{array}$ & $=\frac{\begin{array}{c}\text { Citas recibidas en el año por los artículos } \\
\text { publicados durante los } 2 \text { inmediatamente } \\
\text { anteriores }\end{array}}{\begin{array}{c}\text { Número total de articulos publicados en la } \\
\text { revista en los } 2 \text { años inmediatamente } \\
\text { anteriores }\end{array}}$ \\
\hline
\end{tabular}

\section{INDICADORES BIBLIOMÉTRICOS}

Para lograr los fines de medición del conocimiento producido, la Bibliometría se vale de indicadores que se configuran como rasgos que explican el comportamiento de la ciencia y la tecnología, los cuales son definidos como medidas obtenidas a partir del análisis estadístico de los rasgos cuantificables de la literatura científica (7); su importancia radica en ser instrumentos básicos mediante los cuales la sociedad puede acceder a un conocimiento sobre la estructura real de la ciencia y, en su caso, orientar en lo posible la dirección del desarrollo del conocimiento, además de su empleo para el diseño de políticas científicas con vistas a la evaluación de los sistemas de investigación.

La posibilidad de conocer cuáles son los resultados obtenidos a partir de la adopción de una batería de indicadores que permitan hacer mediciones, resulta fundamental para poder mejorar las políticas futuras y en consecuencia, la calidad de la investigación. A su vez, estas medidas necesitan de un proceso de retroalimentación del sistema y deben ir acompañadas de una evaluación continuada que permita conocer si los objetivos que se persiguen están siendo alcanzados con la mayor eficacia posible (8).

Se puede definir como "indicadores" los parámetros que se utilizan en el proceso evaluativo de cualquier actividad. Normalmente se emplea un conjunto de ellos, cada uno de los cuales pone de relieve una faceta del objeto de la evaluación. Para medir la actividad científica se emplean indicadores bibliométricos, pues permiten analizar el tamaño, el crecimiento y la distribución de la bibliografía, analizar sus procesos de generación, propagación y uso, e identificar a los agentes implicados en su producción y utilización (autores, grupos de investigación, redes de colaboración, organismos, revistas, etc.).

La evaluación de la actividad científica y de la productividad de los investigadores es una cuestión de interés desde muchas perspectivas distintas: la financiación de la investigación, la recompensa de la actividad de investigación, la formulación de políticas de investigación y la toma de decisiones relacionadas con tales políticas, la planificación estratégica de la actividad universitaria, la dotación de plazas de profesorado universitario y su promoción así como la concesión de becas, entre otras. 
Por estas razones, la preocupación por sistemas "justos" de evaluación de la actividad investigadora (como evaluar la creación científica) ha sido y es un preocupación tanto para los propios implicados en la investigación como para aquellos que tienen que tomar decisiones al respecto (9).

\section{Indicadores de productividad científica:}

Estos indicadores (ver tabla 1) se basan en el recuento de publicaciones científicas. Son los más fáciles de utilizar ya que proporcionan información sobre las características de las unidades analizadas. El número de publicaciones es una medida razonada de la actividad científica, pero no del progreso científico.

El cómputo de las publicaciones permite, además, conocer el dinamismo de un área a estudio (según aumente, disminuya o se mantenga) (10). El indicador básico, y a la vez el más sencillo, es la cantidad de publicaciones producida por un autor, país o institución durante un período determinado.

Estos indicadores están condicionados por varios factores; así el cómputo de las publicaciones no proporciona idea de la calidad de éstas, ignora otros métodos no formales de comunicación en ciencia (reuniones, informes, etc.), no tiene en cuenta que las prácticas de publicación evolucionan con el tiempo y que existen presiones políticas y sociales que obligan a publicar (11).

Ahora bien, algunos países intentan valorar la calidad de las publicaciones más que su número. Así, por ejemplo, algunas universidades norteamericanas admiten un número limitado de publicaciones en el curriculum vitae de los aspirantes a plazas de profesor o a los profesores que desean promocionarse a un puesto superior. El mismo criterio existe en España en la evaluación de la actividad investigadora por parte de la Agencia Nacional de Evaluación de la Calidad y Acreditación (ANECA). Pero la corrección de la productividad científica mediante la evaluación de la calidad de las publicaciones es un problema que está lejos de haber sido resuelto ya que difícilmente se puede aplicar un mismo criterio, incluso dentro de las diferentes áreas de las ciencias de la salud.

\section{Indicadores de colaboración:}

Bajo el término "colaboración en ciencia" se incluye una amplia gama de relaciones y actividades conjuntas entre grupos de investigación e instituciones en las que la magnitud y naturaleza de la contribución puede ser muy variable. Podríamos citar la colaboración entre autores que pueden ser del mismo centro, del mismo país, aunque de distintas instituciones, o de países diferentes (12).

Estos indicadores (ver tabla 1) se utilizan para determinar la actividad y cooperación científica habida entre instituciones o grupos científicos, ya que el número de artículos producidos por dichos colectivos es proporcional a su actividad investigadora, y por tanto, se puede considerar como un índice de ésta. Además, la frecuencia relativa del número de trabajos escritos en colaboración entre grupos es proporcional al grado de cooperación científica del grupo y proporciona un indicador de esta cooperación (13).

La naturaleza técnica y compleja de la investigación biomédica, así como la multidisciplinariedad de alguna de sus áreas, requiere la contribución de varios profesionales, es decir, el trabajo en equipo. Este trabajo se reconoce por medio de la inclusión como autores en las publicaciones a todos los investigadores que han participado en el trabajo (10).

Miden las relaciones que han existido entre los productores o agentes científicos y que han finalizado con la publicación conjunta de resultados científicos. Se basan en los datos de autoría. En Bibliometría se sigue el concepto de colaboración internacional como un indicador de globalización. 
El indicador más utilizado para referirse al grado de colaboración es el índice firmas/trabajo, también denominado índice de coautoría o índice de colaboración. Este indicador puede estar sobrestimado por la llamada "autoría honoraria" (personas de relevancia académica que figuran como autores sin haber participado en el estudio), o por la "autoría de agradecimiento" (personal de apoyo técnico incluido en el trabajo sin cumplir los criterios de autoría). Pero también puede estar "infraestimado" por darse el caso de la "autoría fantasma" (experto que colabora de forma muy directa en el estudio, participa en la fase conceptual del diseño, en el apartado metodológico e incluso en la redacción del manuscrito final y no figura como autor del artículo).

\section{Indicadores de circulación:}

Se sustentan en razón de que los resultados científicos deben circular por canales formales que hagan posible la socialización del conocimiento entre la comunidad científica. Miden la presencia de documentos en las bibliotecas y bases de datos bibliográficas.

Están condicionados por la especialización y el idioma de las publicaciones. Estos indicadores se utilizan para ver la distribución de las revistas y su cobertura en las bases de datos. Los principales indicadores se muestran en la tabla 1.

En ciencias de la salud, al existir varias bases de datos bibliográficas que se complementan, infieren a estos indicadores una buena validez y fiabilidad. La comunicación de los documentos a través de Internet permite que las comunidades académicas y sociales conozcan la producción científica que se está generando en diferentes partes del mundo. Y en consecuencia se traduce en ventajas de "visibilidad" de los investigadores y sus productos científicos.

Asimismo, al tratarse de la circulación del conocimiento científico, de su expansión y visibilidad, se está ante factores intangibles, que gracias a los indicadores bibliométricos se cuantifican y permiten estudiar e identificar diferentes aspectos de la actividad investigadora, como áreas temáticas prioritarias, disciplinas emergentes, redes de excelencia o el grado de relevancia de las instituciones.

\section{Indicadores de consumo:}

Los análisis sobre el consumo de información científica se basan en el estudio de citas y referencias (ver tabla 1).

Una cita es una transacción científica, un reconocimiento expreso de una deuda intelectual hacia una fuente de información previa (14). Y, en consecuencia, se puede definir:

- Cita: mención textual o factual que se hace de otro documento en el texto que se redacta.

- Referencia: dato bibliográfico que se ofrece para identificar de modo preciso la fuente mencionada.

También se puede distinguir entre "citas" (que una publicación recibe de otras posteriores) y "referencias" (que una publicación hace de otras anteriores) (15).

El proceso de citación es obligatorio en la ciencia actual ya que la normativa científica moderna requiere que cuando un autor publica los resultados de un trabajo, se refiera a los que lo han precedido. Estas referencias conducen a las fuentes de las ideas contenidas en el trabajo y su función es reconocer que los métodos, interpretaciones, datos, etc., que se citan proceden de los autores y publicaciones citadas. A partir del análisis de citas y referencias se puede determinar el consumo de información científica de los autores, instituciones, revistas, etc., así como la repercusión que su producción ha tenido en comunidades científicas determinadas. El análisis de citas también permite seguir la pista del desarrollo intelectual y científico de las nuevas disciplinas emergentes (10). 
Ya se sabe que el consumo de la información se estudia mediante el análisis de las referencias. De los numerosos e importantes indicadores que proporciona, dos interesan especialmente a la evaluación: los correspondientes a la obsolescencia y al aislamiento (16).

Estos indicadores se apoyan en el supuesto de que los trabajos importantes son usualmente citados, mientras que los irrelevantes se ignoran, si bien la realidad es mucho más compleja. Es patente que el mayor aislamiento de la producción científica de países como Japón y la Unión Soviética, en contraste con lo que sucede con la de los Estados Unidos y la mayoría de los países de la Europa occidental, está estrechamente ligado al idioma, independientemente de la calidad y pertinencia de su producción científica.

\section{Indicadores de repercusión e impacto:}

Estos indicadores (ver tabla 1) proceden, también, del análisis de citas y referencias, y, al igual que los indicadores de consumo, se apoyan en el supuesto de que los trabajos importantes son usualmente más citados.

Se basan en los recuentos del número de citaciones que reciben los documentos durante un periodo determinado de tiempo o a partir de su publicación. El principal indicador, destacando sobre el resto, para la evaluación de la calidad de la producción científica recogida en una determinada revista, es el "Factor de Impacto" (ver figura 5). Es una medida de la frecuencia con la que el artículo medio de una revista ha sido citado en un período. Básicamente mide la relación entre las citas recibidas y los artículos publicados en una revista.

Garfield (17), en 1964, creó el Science Citation Index, estableciendo el "famoso" indicador ya nombrado: el factor de impacto. Supone la primera medida objetiva, cuantificable y estable de la valoración de una revista en el ámbito científico y, por ello, ha ido ganando aceptación por parte de la comunidad científica, si bien no está exenta de inconvenientes.

Al presente, el Factor de Impacto se publica anualmente (mes de julio) en el Journal Citation Reports (JCR), a partir de los datos del Science Citation Index Expanded y de los datos de la Social Citation Index y de Arts \& Humanities Citation Index; ver tabla 1.

\section{LIMITACIONES DE LA BIBLIOMETRÍA}

La evaluación de la Ciencia a duras penas se concibe ya sin el recurso de los métodos bibliométricos; su estilo se ha depurado y matematizado hasta el extremo de que algún autor ha llegado a decir que se ha convertido en una hard science (18). Sin embargo, sus aspectos conceptuales están sometidos a un perpetuo proceso de reelaboración en el que los especialistas parecen incapaces de ponerse de acuerdo.

En el informe de expertos del Comité de Naciones Unidas del año 1984 (19) se manifestaba que existía una falta de base teórica para el desarrollo y análisis de indicadores, se ponía en duda la total validez y veracidad de los indicadores, se llamaba la atención acerca del problema que supone el comentar la colección de todos los datos necesarios y se relataba el alto costo que suponía estos estudios.

Por ejemplo, los "Indicadores de productividad científica" son efectivos en aquellas áreas en las que las publicaciones son un resultado esencial de la investigación. Por esta razón, su validez es de máxima relevancia en el estudio de las áreas básicas, donde predominan publicaciones científicas, y menor en las áreas tecnológicas o aplicadas (20). Además, si para medir el crecimiento del conocimiento, es este el indicador a emplear, hay que asumir: que todo el conocimiento obtenido por los 
científicos se encuentra en estos trabajos y que cada uno de los trabajos contiene igual proporción de conocimiento. Siendo obvio que ninguna de estas dos afirmaciones es cierta.

A esto habría que añadir los defectos de las bases de datos bibliográficas que se utilizan para recabar estos datos y que comportan numerosos errores que deberían ser eliminados antes de construir indicadores fiables. Habría que normalizar los contenidos de algunos campos documentales (nombre de instituciones, de las revistas, de los países, etc.) que habitualmente no se encuentran normalizados en las principales bases de datos utilizados como fuente para elaborar los indicadores bibliométricos. Tener conocimiento de las obras de referencia existentes es condición necesaria para resolver cualquier problema informativo que se plantee en el curso del trabajo bibliométrico.

Por otro lado, estos indicadores, como ya se ha mencionado, no valoran la calidad de las publicaciones, ignoran otros métodos no formales de comunicación en ciencia (reuniones, informes, etc.), no tienen en cuenta que las prácticas de publicación evolucionan con el tiempo y, asunto importante, existen presiones políticas y sociales que obligan a publicar.

La consideración del número de publicaciones como indicador de actividad científica ha desencadenado el denominado "síndrome de publicar o perecer" (síndrome POP o publish or perish). Bajo este nombre se designa la situación actual en la que los científicos se ven presionados a publicar, no solo para dar a conocer los resultados de su investigación, sino también como la única vía de justificar su actividad y obtener reconocimiento. En esta misma línea se han descrito otros comportamientos como son la denominada "autoría gratuita" y la fragmentación de los trabajos en varias publicaciones que podrían haberse publicado en un solo artículo más completo y más coherente (síndrome LPU o least publishable unit). En España se conoce como "publicación salami" $(22,23)$.

En relación a los "Indicadores de colaboración" se conoce que sus datos pueden estar sobreestimados o por el contrario infraestimados, dependiendo de la inclusión o no, de determinados autores.

Así, este indicador puede estar sesgado al constituirse en torno a los autores con elevada productividad los llamados "colegios invisibles" que van a favorecer una proyección de los autores con menos predicamento (según la ley de distribución hiperbólica de ventaja acumulativa, el éxito genera éxito).

De igual forma, es difícil establecer una adecuada correlación entre el orden de firmas y la producción científica, lo que supone una grave limitación. En esta línea, señalar que algunas bases de datos bibliográficas tienen la particularidad de indizar solo el nombre e institución del primer autor o del que figura como autor de correspondencia, situación que complica el estudio de este indicador (24).

En cuanto a los "Indicadores de circulación" hay que tener en cuenta que están condicionados por la especialización y el idioma de las publicaciones, dependiendo fundamentalmente de las bases de datos bibliográficas. Está probado que las áreas básicas y clínicas están mejor representadas en las bases que recogen literatura científica sobre ciencias de la salud -MEDLINE, EMBASE, etc.- que las áreas aplicadas o técnicas.

Los "Indicadores de consumo, repercusión e impacto", basados en las citas bibliográficas, se han empleado, por desgracia, de forma indiscriminada y al margen de toda cautela crítica (16). Dicho empleo acrítico no tiene en cuenta, en primer lugar, las advertencias del propio Eugene Garfield (17) (precursor de estos indicadores), que ha repetido innumerables veces que es un indicador relativo, que no debe aplicarse para comparar revistas, grupos o autores de disciplinas distintas. En segundo lugar, ignora el debate en torno a las funciones de las citas en la comunicación científica que son muy complejas y en absoluto sirven de fundamento a interpretaciones simples.

Garfield (25) declaraba, más de 50 años después de la creación del Factor de Impacto, en el International Congress on Peer Review and Biomedical Publication: 
En 1955, no se me ocurrió que el 'impacto' podría llegar a ser cuestionable. Al igual que la energía nuclear, el factor de impacto es una bendición mixta. Yo esperaba que se usara de forma constructiva, pero a la vez, me daba cuenta de que en manos equivocadas, podría ser utilizado abusivamente.

Posteriormente, en la publicación de esta conferencia (26), concluía:

El uso del impacto de las revistas para evaluar a los individuos contiene peligros inherentes.

A medida que se investiga más en el fenómeno de la citación, menos se entiende por qué se cita un determinado trabajo y no otro. Se indica que, aunque las citas se puedan contar muy fácilmente, no sabemos muy bien qué es lo que se está midiendo. En este sentido, hay que tener en cuenta que menos de una tercera parte de las fuentes citadas son consideradas esenciales para los que las citan (27).

Es difícil predecir en la citación qué proporción se debe a la calidad intrínseca del trabajo citado y cuánto a otros factores como prestigio de la revista citada o prestigio de la institución a la que pertenece el autor, ya que hay que considerar que el fenómeno de la citación está sujeto a modas, fobias, y otras tendencias (13). Por ende, el número de citas que recibe un trabajo no es una medida de su calidad científica, sino que más bien indica su visibilidad, uso, difusión o impacto.

En este punto, es interesante destacar algunos inconvenientes que supone el empleo de las citas en los estudios bibliométricos:

1. Problemas conceptuales: El principal obstáculo del uso de las citas es la ausencia de un modelo aceptado que explique el proceso de citación. Los autores citan aquellos trabajos que han influido en su investigación, pero algunos estudios revelan que no se citan todas las influencias y que, sin embargo, se incluyen trabajos que no se han utilizado realmente en la investigación (28).

En el proceso de citación intervienen varios elementos:

- Un sistema recompensador por el que se agradece la aportación hecha por el autor citado.

- Un sistema retórico, por el que se trata de convencer de las tesis mantenidas.

- Un sistema de comunicación, por el que se tienden a utilizar revistas de alto prestigio y visibilidad que apoyan la credibilidad del trabajo propio.

2. Problemas técnicos: El uso de las citas queda actualmente limitado a las bases de datos bibliográficas. Algunos problemas observados en el uso de estas bases de datos incluyen, entre otros: errores tipográficos en los nombres de los autores o en la referencia bibliográfica, existencia de homónimos o presencia de autores que firman con distintos nombres a lo largo del tiempo.

3. Diferencias entre tipos documentales: El tipo de documento puede influir sobre el número de citas que reciben las publicaciones. Se han descrito altas tasas de citación para los documentos metodológicos, que introducen técnicas o métodos que se referencian luego en cada uso. Asimismo, las revisiones y, por extensión, las revistas especializadas en revisiones obtienen tasas de citación más altas que los otros tipos documentales debido a que manejan una amplia bibliografía y su consulta es especialmente útil para los científicos.

4. Diferencias entre áreas: Son inadecuadas las comparaciones entre áreas basadas en el número de citas o en el factor de impacto. La razón es que existen importantes diferencias en el número de citas que pueden recibir los trabajos según las áreas científicas a causa de las diferencias 
en el tamaño de las comunidades científicas, los hábitos de citación y el ritmo de envejecimiento de la bibliografía.

La frecuencia media de citación en una materia depende del número medio de referencias por artículo en dicha materia, valor determinado en parte por el tamaño del área y sus hábitos de citación. Por otro lado, la probabilidad de que los trabajos sean citados es mayor en áreas grandes y generales, por ejemplo, bioquímica, que en áreas pequeñas o minoritarias, como micología, que difícilmente alcanzarán altas tasas de citación por el menor número de posibles científicos interesados.

Como ejemplo de la diferencia entre áreas se puede señalar que solo un 5\% de los documentos de arte y humanidades reciben alguna cita en los 5 años posteriores a su publicación, frente al $25 \%$ de los documentos en ciencias sociales y el 30-40\% en ingeniería y tecnología. En el caso de medicina, se ha descrito que un 50-60\% de los documentos reciben citas a los 5 años de su publicación (29).

Es sabido que las citaciones que alcanza la investigación básica siempre son superiores a las de la investigación clínica y epidemiológica. También se conoce que los artículos de revisión son los que tienen mayor oportunidad de ser citados y actualmente, las revisiones sistemáticas al reunir la evidencia publicada sobre un tema puntual.

Las limitaciones expuestas para el análisis de citas son también válidas para el uso del factor de impacto ya que este indicador se calcula en función de las citas que reciben las revistas.

Al igual que ocurría con las citas, el factor de impacto de las revistas también muestra valores muy distintos según las áreas. Tan solo pone de manifiesto las diferencias en el número de citas que se reciben en las distintas áreas, hecho en el que influyen, como ya se señaló anteriormente, el tamaño de la comunidad científica, el ritmo de envejecimiento de la bibliografía y los hábitos de citación. Las áreas más clínicas presentan los factores de impacto más bajos, mientras que los factores de impacto más elevados corresponden a áreas más básicas, como la bioquímica y la biología molecular o la inmunología. Evidentemente, las áreas de ciencias aplicadas y técnicas son escasamente recogidas en los listados bibliográficos de los trabajos científicos.

Las áreas con un rápido envejecimiento de la bibliografía presentan valores altos de factor de impacto. Esto es así debido a que se calcula el factor de impacto en función de las citas recibidas durante los dos años siguientes a la publicación de los documentos (impacto a corto plazo). En las áreas de rápido envejecimiento (por ejemplo la biología molecular o la genética) se citan sobre todo documentos muy recientes, y todas estas citas se considerarán en el cálculo del factor de impacto. Por el contrario, un alto porcentaje de las citas que reciben las revistas de áreas de lento crecimiento (como cirugía o pediatría) tendrán una antigüedad mayor de 2 años y no se considerarán en el cálculo del factor de impacto. Para analizar estas últimas revistas sería más adecuado utilizar una ventana de citación más amplia, por ejemplo, de 4 o 6 años (impacto a medio plazo). En este momento, el propio JCR recoge el factor de impacto de 5 años.

Por otro lado, el uso del número de citas como indicador del impacto o visibilidad de las publicaciones conlleva el riesgo de que se produzca un proceso de "citación dirigida" (30).

Conscientes del valor otorgado a las citas, algunos investigadores intentan aumentar su tasa de citación a través de la "autocitación" o de acuerdos de citación mutua con otros investigadores o grupos. En cualquier caso, la autocitación por sí misma no puede alterar de forma significativa el número de citas que reciben los documentos y cierto grado de autocitación es lógico, ya que la investigación es un proceso acumulativo y los resultados actuales de la investigación suelen ser el punto de partida para nuevos avances. Es interesante señalar cómo los propios editores de las 
revistas, conocedores del interés de las citas para aumentar la visibilidad de su revista, intentan de forma dirigida fomentar la autocitación de la revista en los documentos que publican (31).

Como corolario a este apartado se podía manifestar que los indicadores bibliométricos se ven como una herramienta rentable, principalmente porque dan información cuantitativa "concentrada" sobre la producción (output) y el impacto de esa producción. Al mismo tiempo, y a pesar de posibles limitaciones, los indicadores bibliométricos juegan un papel palmario en la toma de decisiones en política científica y en evaluaciones del rendimiento de la investigación y hay una considerable evidencia de que los indicadores bibliométricos desempeñan un importante papel como herramienta de evaluación de los grupos de investigación o de los científicos individuales.

En consecuencia, dadas las repercusiones que las evaluaciones bibliométricas tienen sobre la asignación de fondos para la investigación e incluso la acreditación y promoción profesional de los investigadores, es necesario conocer muy bien las particularidades y las limitaciones que su uso conlleva.

Tabla 1: Indicadores bibliométricos y su definición

\begin{tabular}{|c|c|}
\hline \multicolumn{2}{|l|}{ Indicadores de productividad científica } \\
\hline Índice de actividad & Porcentaje de producción (de una zona, institución, etc.) \\
\hline Índice de número de artículos/habitante & $\begin{array}{l}\text { Cociente entre el número de artículos producidos y la población } \\
\text { de una zona }\end{array}$ \\
\hline Índice de número de artículos/PIB & $\begin{array}{l}\text { Cociente entre el número de artículos producidos y el Producto } \\
\text { Interior Bruto(PIB) de una zona }\end{array}$ \\
\hline Índice publicaciones/periodo & $\begin{array}{l}\text { Número de publicaciones producidas en un periodo (mes, año, } \\
\text { quinquenio, etc.) }\end{array}$ \\
\hline Índice de producción & Número de artículos producidos por un autor \\
\hline Índice de productividad o de Lotka & Logaritmo del número de artículos publicados \\
\hline Índice de productividad fraccionada & $\begin{array}{l}\text { Logaritmo del sumatorio de } 1 / n \text {, siendo n el número de firmantes } \\
\text { de cada trabajo de un autor }\end{array}$ \\
\hline Índice de transitoriedad & $\begin{array}{l}\text { Porcentaje de autores que solo han publicado un trabajo sobre } \\
\text { un tema }\end{array}$ \\
\hline Intervalo aceptación - publicación & $\begin{array}{l}\text { Número de días transcurridos entre la aceptación de un artículo } \\
\text { por un revista y su publicación }\end{array}$ \\
\hline Intervalo recepción - aceptación & $\begin{array}{l}\text { Número de días transcurridos entre la recepción de un artículo } \\
\text { por un revista y su aceptación }\end{array}$ \\
\hline Intervalo recepción - publicación & $\begin{array}{l}\text { Número de días transcurridos entre la recepción de un artículo } \\
\text { por un revista y su publicación }\end{array}$ \\
\hline Tamaño bibliométrico & $\begin{array}{l}\text { Número de publicaciones producidas por una autor, institución, } \\
\text { país, etc. }\end{array}$ \\
\hline
\end{tabular}




\begin{tabular}{|c|c|}
\hline Tasa de referencias/artículo & $\begin{array}{l}\text { Cociente entre el número de referencias emitidas y el número de } \\
\text { artículos publicados }\end{array}$ \\
\hline \multicolumn{2}{|l|}{ Indicadores de colaboración } \\
\hline $\begin{array}{l}\text { Índice de coautoría (Número de firmas } \\
\text { por trabajo) }\end{array}$ & Cociente entre el número de autores y el número de artículos \\
\hline Índice de colaboración institucional & $\begin{array}{l}\text { Cociente entre el número de centros firmantes y el número de } \\
\text { artículos }\end{array}$ \\
\hline Tasa de colaboración nacional & $\begin{array}{l}\text { Porcentaje del número de artículos de colaboración nacional } \\
\text { sobre la producción de un país }\end{array}$ \\
\hline Tasa de colaboración internacional & $\begin{array}{l}\text { Porcentaje del número de artículos de colaboración internacional } \\
\text { sobre la producción de un país }\end{array}$ \\
\hline \multicolumn{2}{|l|}{ Indicadores de circulación } \\
\hline Índice de productividad circulante & $\begin{array}{l}\text { Logaritmo del número de trabajos incluidos en una base de datos } \\
\text { bibliográfica }\end{array}$ \\
\hline Índice de circulación & $\begin{array}{l}\text { Cociente entre el número de trabajos circulantes y el número de } \\
\text { trabajos publicados }\end{array}$ \\
\hline Índice de difusión internacional & $\begin{array}{l}\text { Cociente entre el número de trabajos circulantes y el número } \\
\text { de trabajos publicados, multiplicado por el número de bases de } \\
\text { datos bibliográficas en las que circulan }\end{array}$ \\
\hline \multicolumn{2}{|l|}{ Indicadores de consumo } \\
\hline Índice de aislamiento (insularity) & $\begin{array}{l}\text { Porcentaje de referencias que corresponden al mismo país que } \\
\text { la publicación citadora }\end{array}$ \\
\hline $\begin{array}{l}\text { Índice de Burton-Kebler o Semiperiodo } \\
\text { (half-life) de las referencias }\end{array}$ & $\begin{array}{l}\text { Mediana de la distribución de las referencias por año de } \\
\text { procedencia }\end{array}$ \\
\hline Índice de Price & Porcentaje de referencias con menos de 5 años \\
\hline \multicolumn{2}{|l|}{ Indicadores de repercusión e impacto } \\
\hline Citas recibidas & Número de citas recibidas por un autor, revista, artículo, etc. \\
\hline Factor de impacto & $\begin{array}{l}\text { Cociente entre el número de citas que han recibido en un año } \\
\text { determinado los documentos publicados en una revista en los } 2 \\
\text { años anteriores y el número de documentos (citables) publicados } \\
\text { por la revista en esos } 2 \text { años }\end{array}$ \\
\hline $\begin{array}{l}\text { Factor de impacto corregido sin } \\
\text { autocitas }\end{array}$ & Factor de impacto calculado sin contar las autocitas \\
\hline
\end{tabular}




\begin{tabular}{|c|c|}
\hline Factor de impacto de 5 años & $\begin{array}{l}\text { Cociente entre el número de citas que han recibido en un año } \\
\text { determinado los documentos publicados en una revista en los } 5 \\
\text { años anteriores y el número de documentos (citables) publicados } \\
\text { por la revista en ese quinquenio }\end{array}$ \\
\hline Factor de impacto de una disciplina & $\begin{array}{l}\text { Cociente entre el número de citas en las revistas principales de } \\
\text { una disciplina en un periodo de tiempo y el número de artículos } \\
\text { publicados en ese periodo }\end{array}$ \\
\hline Factor de impacto esperado & $\begin{array}{l}\text { Factor de la revista en la que se publica un trabajo, aplicado a } \\
\text { dicho trabajo }\end{array}$ \\
\hline Factor de impacto medio por temas & $\begin{array}{l}\text { Media del factor de impacto de las revistas de un área } \\
\text { determinada }\end{array}$ \\
\hline Factor de impacto observado & $\begin{array}{l}\text { Número de citas recibidas por un documento en un periodo de } \\
\text { tiempo determinado }\end{array}$ \\
\hline $\begin{array}{l}\text { Factor de impacto ponderado por } \\
\text { especialidad }\end{array}$ & $\begin{array}{l}\text { Cociente entre el factor de impacto de cada revista y el factor } \\
\text { de impacto máximo de su área (que corresponde al de la revista } \\
\text { con mayor factor de impacto) }\end{array}$ \\
\hline Factor de impacto relativo & $\begin{array}{l}\text { Cociente entre el impacto de una autor (o grupo de autores) en } \\
\text { una disciplina y el impacto de esa disciplina en un país (o grupo } \\
\text { de países) }\end{array}$ \\
\hline Factor de impacto relativo observado & $\begin{array}{l}\text { Cociente entre el número de citas por artículo recibidas por un } \\
\text { país en un determinado tema y el promedio de citas por artículo } \\
\text { en ese tema }\end{array}$ \\
\hline Factor de popularidad & $\begin{array}{l}\text { Cociente entre el número de fuentes que citaron a la revista en un } \\
\text { periodo de tiempo y el número de fuentes citadas por la revista } \\
\text { en ese periodo de tiempo }\end{array}$ \\
\hline Factor de prestigio & $\begin{array}{l}\text { Cociente entre el número de citas que reciben en un año los } \\
\text { artículos originales publicados en una revista en el mismo año y } \\
\text { en los } 2 \text { años anteriores y el número de artículos publicados en } \\
\text { esa revista en esos } 3 \text { años, convertido en una escala de } 0 \text { a } 1000\end{array}$ \\
\hline Factor o índice $\mathrm{H}$ (número de Hirch) & $\begin{array}{l}\text { Número que se asigna a un autor igual al número de artículos } \\
\text { publicados por ese autor que han recibido tantas o más citas } \\
\text { que su factor } \mathrm{H} \text {. }\end{array}$ \\
\hline Índice de citas/artículo & $\begin{array}{l}\text { Cociente entre el número de citas recibidas y el número de } \\
\text { artículos publicados por un autor, revista, país, etc. }\end{array}$ \\
\hline Índice de atracción de una tema & $\begin{array}{l}\text { Cociente entre el porcentaje de citas recibidas por un país } \\
\text { correspondientes a un tema y el porcentaje total de las citas que } \\
\text { corresponden a ese tema }\end{array}$ \\
\hline Índice de autocitas de autores & $\begin{array}{l}\text { Porcentaje de autocitas de autores con respecto al total de citas } \\
\text { recibidas }\end{array}$ \\
\hline
\end{tabular}




\begin{tabular}{|c|c|}
\hline Índice de autocitas de la revista & $\begin{array}{l}\text { Porcentaje de autocitas de la revista con respecto al total de } \\
\text { citas recibidas }\end{array}$ \\
\hline Índice de citación & $\begin{array}{l}\text { Cociente entre el número de citas recibidas por una revista en un } \\
\text { periodo de tiempo y el número de referencias aportadas por la } \\
\text { revista en ese periodo de tiempo. }\end{array}$ \\
\hline Índice de impacto & $\begin{array}{l}\text { Cociente entre el número de citas recibidas y el número de } \\
\text { trabajos publicados }\end{array}$ \\
\hline Índice de influencia & $\begin{array}{l}\text { Cociente entre el número de citas recibidas y el número de } \\
\text { referencias emitidas }\end{array}$ \\
\hline Índice de inmediatez & $\begin{array}{l}\text { Número de citas que recibieron los artículos de una revista en un } \\
\text { año determinado dividido entre el número total de artículos que la } \\
\text { revista publicó en ese mismo año }\end{array}$ \\
\hline $\begin{array}{l}\text { Índice de investigación potencial } \\
\text { realizada }\end{array}$ & $\begin{array}{l}\text { Número de citas recibidas por los artículos de una revista en } \\
\text { un periodo de tiempo dividido entre el número de artículos } \\
\text { aparecidos en la revista en ese periodo de tiempo }\end{array}$ \\
\hline Índice de visibilidad & Logaritmo de las citas recibidas \\
\hline Índice de visibilidad esperado & $\begin{array}{l}\text { Índice de visibilidad de la revista en la que se publica un trabajo, } \\
\text { aplicado a dicho trabajo }\end{array}$ \\
\hline Influencia ponderada & $\begin{array}{l}\text { Cociente entre el número de citas de la revista en otras revistas y } \\
\text { el número de referencias de la revista en otras revistas }\end{array}$ \\
\hline Nivel de impacto & $\begin{array}{l}\text { Cociente entre el factor de impacto real y el factor de impacto } \\
\text { esperado }\end{array}$ \\
\hline Número total ajustado a citas & Cociente entre el número de citas y el número de autores citados \\
\hline $\begin{array}{l}\text { Número (porcentaje) de artículos } \\
\text { citados }\end{array}$ & $\begin{array}{l}\text { Número o porcentaje sobre el total de artículos citados de una } \\
\text { revista, autor, etc. }\end{array}$ \\
\hline Número de artículos clave & Número de artículos citados más de 15 veces en una año \\
\hline Número de autores citadores & $\begin{array}{l}\text { Número de autores que han citado a una revista, región, país, } \\
\text { etc. }\end{array}$ \\
\hline Número de autores citados & Número de autores citados de una revista, región, país, etc. \\
\hline Número de descubrimientos cruciales & Número de artículos citados más de 100 veces en un año \\
\hline Percentil de la distribución de citas & $\begin{array}{l}\text { Percentil que ocupa un autor en la distribución de citas de una } \\
\text { revista, área temática, etc. }\end{array}$ \\
\hline & \\
\hline
\end{tabular}




\section{BIBLIOGRAFÍA}

1. Tomás Casterá VJ. Estudio bibliométrico de la producción científica y de consumo de las revistas sobre nutrición indizadas en la Red SciELO [tesis doctoral]. Alicante, España: Universidad de Alicante; 2013.

2. Price DJS. Little science, big science. New York: Columbia University Press; 1963. [Consultado edición en español: Hacia una ciencia de la ciencia. Barcelona, España: Ariel; 1973.].

3. Garfield E. Citation indexes for science: a new dimension in documentation through association of ideas. Science. 1955;122:108-11. DOI: 10.1126/science.122.3159.108; PMID: 14385826

4. Garfield E. Citation indexing for studying science. Nature. 1970;227:669-71. PMID: 4914589

5. Garfield E. Is citation analysis a legitimate evaluation tool?. Scientometrics. 1979;1(4):359-75.

6. Price DJS. Networks of scientific papers. Science. 1965; 149: 510-15. DOI: 10.1126/science.149.3683.510

7. Maltras-Barba B. Indicadores Bibliométricos. Fundamentos y aplicación al análisis de la ciencia. Gijón, España: Ediciones Trea; 2003.

8. Corera Álvarez E, Gómez-Crisóstomo MR, González-Molina A, Muñoz Fernández FJ, Vargas Quesada B. Indicadores Bibliométricos de la Actividad Científica Española (1990-2004). De Moya Anegón F, dirección, Chinchilla Rodríguez Z, coordinación. Madrid, España: Fundación Española para la Ciencia y la Tecnología; 2005.

9. Páez Rovira D, Salgado Velo JF. Indicadores de productividad científica: implicaciones para la evaluación de la psicología española. Boletín de Psicología. 2009;(97):117-36.

10. Aleixandre R. Bibliometría e indicadores de actividad científica. En: Jiménez Villa J, Argimon PaIlàs JM, Martín Zurro A, Vilardell Tarrés. Publicación científica biomédica: como escribir y publicar un artículo de investigación. Barcelona, España: Editorial Elsevier España; 2010. p. 363-84.

11. Sanz-Valero J, Wanden-Berghe C. Análisis bibliométrico de la producción científica, indizada en MEDLINE, sobre los servicios de salud proporcionados por las unidades de hospitalización a domicilio. Hosp Domic. 1(1);2017:21-34. DOI: 10.22585/hospdomic.v1i1.3

12. Sancho R, Morillo F, De Filippo D, Gómez I, Fernández MT. Indicadores de colaboración científica inter-centros en los países de América latina. Interciencia. 2006;31(4):284-92.

13. Sancho R. Indicadores Bibliométricos utilizados en la evaluación de la ciencia y la tecnología: Revisión Bibliográfica. Rev Esp Doc Cient. 1990:13(3-4):842-65.

14. Culebras JM, García de Lorenzo A, Gil A, Laborda L, Pérez de la Cruz A, Sánchez Álvarez C. Las referencias bibliográficas en la nutrición. Sanz-Valero J, Wanden-Berghe C, coordinadores. Madrid, España: Grupo de Comunicación y Documentación Científica en Nutrición (CDC-Nut SENPE); 2007.

15. Krauze TJ, Hillinger C. Citation, references and the growth of scientific literature: A model of dynamic interaction. J Am Soc Inf Sci. 1971;22(5):332-6. DOI: 10.1002/asi.4630220507

16. López Piñero JM, Terrada ML. Los indicadores bibliométricos y la evaluación de la actividad médico-científica (III): Los indicadores de producción, circulación y dispersión, consumo de la información y repercusión. Med Clin (Barc). 1992;98(4):142-8.

17. Garfield E. Citation indexing. Its theory and application in science, technology and humanities. Nueva York, USA: Wiley Editors, 1979. 
18. Wouters PLL. Has Price's dream come true? Is scientometrics a hard science? Scientometrics. 1994;31(2):193-222.

19. United Nations advisory committee on science and technology for development. Indicators of measurement of impact for development. Scientometrics. 1984;6(6):449-63.

20. Camps D. Limitaciones de los indicadores bibliométricos en la evaluación de la actividad científica biomédica. Colombia Médica. 2008;39(1):74-9.

21. Garfield E. What Is The Primordial Reference For The Phrase 'Publish Or Perish'? The Scientist. 1996;10(12):11.

22. Broad WJ. The publishing game: getting more to less. Science. 1981;211(4487):1137-9.

23. Castiel LD, Sanz-Valero J. Entre fetichismo e sobrevivência: o artigo científico é uma mercadoria acadêmica? Cad Saúde Pública. 2007;23(12):3041-50. DOI: 10.1590/S0102311X2007001200026; PMID: 18157347

24. De Filippo D, Morillo F, Fernández MT. Indicadores de colaboración científica del CSIC con Latinoamérica en bases de datos internacionales. Rev Esp Doc Cient. 2008;31(1):66-84. DOI: 10.3989/redc.2008.v31.i1.413

25. Garfield E. The Agony and the Ecstasy: the history and meaning of the journal Impact Factor. In: Fifth International Congress on Peer Review and Biomedical Publication. Chicago, USA: Thomson Reuters; 2005.

26. Garfield E. The history and meaning of the journal Impact Factor. JAMA. 2006;295(1):90-3. DOI: 10.1001/jama.295.1.90; PMID: 16391221

27. González de Dios J, Moya JM, Mateos Hernández M A. Indicadores bibliométricos: Características y limitaciones en el análisis de la actividad científica. An Esp Pediatr. 1997;47(3):235-44

28. Cozzens SE. What do citations counts? The rethoric-first model. Scientometrics. 1989;15(5-6): 437-47.

29. Hamilton DP. Research papers: who's uncited now? Science. 1991; 251(4989): 25. DOI: 10.1126/ science.1986409; PMID: 1986409

30. Bordons M, Zulueta MA. Evaluación de la actividad científica a través de indicadores bibliométricos. Rev Esp Cardiol. 1999;52(10):790-800. DOI: 10.1016/S0300-8932(99)75008-6

31. Castiel LD, Sanz-Valero J. Política científica: manejar la precariedad de los excesos y desnaturalizar la ideología "publicacionista" todopoderosa. Salud Colectiva. 2009;5(1):5-11. 